\title{
Designer surface plasmon dispersion on a one-dimensional periodic slot metasurface with glide symmetry
}

\author{
Miguel Camacho ${ }^{1,}$, Rhiannon C. Mitchell-Thomas ${ }^{1}$, Alastair P. Hibbins ${ }^{1}$, J. Roy Sambles ${ }^{1}$, \\ AND Oscar Quevedo-Teruel ${ }^{2}$ \\ ${ }^{1}$ Electromagnetic and Acoustic Materials Group, Department of Physics and Astronomy, University of Exeter, Stocker Road, Exeter EX4 4QL, United \\ Kingdom \\ ${ }^{2}$ Department of Electromagnetic Engineering, KTH Royal Institute of Technology, Stockholm, Sweden \\ *Corresponding author: mc586@exeter.ac.uk
}

The existence of bound surface modes, surface plasmon polaritons supported at optical frequencies by metal-dielectric interfaces is well established and has been studied since the mid nineteenth century [1]. Since then, the ability to control such bound waves has attracted much attention given their wide range of applications varying from imaging to plasmonic communication devices [2,3]. At lower frequencies, in the microwave regime, adding structure to metal surfaces allows analogous surface modes, known as spoof or designer surface plasmons, to be supported [4-6]. This allows for twodimensional microwave devices, such as lenses or antennas to control surface wave propagation $[7,8]$. In the recent years, the study of these low-frequency surface modes has also benefited from the development of ultra-thin flexible metasurfaces with important applications to wearable devices $[9,10]$.

In general surface modes present a strongly frequencydependent effective refractive index, which limits the frequency band over which these two dimensional devices, commonly known as metasurfaces, maintain their designed behavior. The ability to engineer the frequency dependence of the mode index has motivated much literature [11-13] and has led to the exploration of diverse symmetries [14] and geometries to control the confinement of the fields [15].

In the recent years, the use of glide symmetry has provided a route towards increasing the frequency bandwidth of such devices. Glide-symmetric geometries are periodic structures that are invariant under a combination of translation and reflection. This symmetry allows for a near linear dispersion relation thanks to a degeneracy with non-zero group velocity of the supported modes at the Brillouin zone boundary [16, 17]. The properties of glide symmetry were first studied in the 1960s [18, 19], although they had been little exploited until the development of metasurfaces, providing a design tool for broadband lenses [20] and electromagnetic band gap structures [21].

In this Letter we present a study of glide symmetry for a single-layered, one dimensional periodic structure. Specifically, the structure under consideration is a negligible thickness infinite metal layer, perforated with an infinitely-long slot. This slot is periodically notched on both sides so that the system

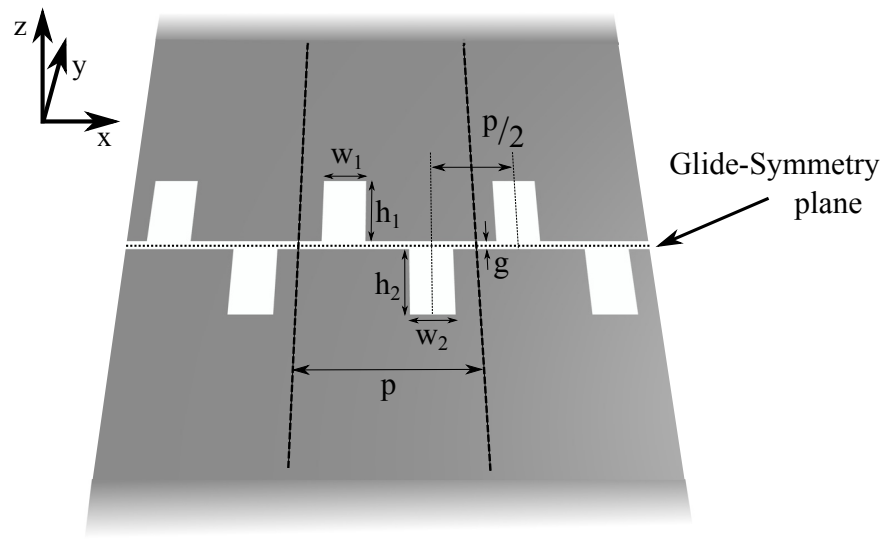

Fig. 1. Perspective view of the infinitely-long double notched slot in a glide symmetric configuration. The dashed line represents the plane used for the mirror operation required by the glide symmetry.

possesses glide symmetry (for which the two sub-lattices of notches are displaced by half of their respective unit cell) as shown in Fig. 1. For the sake of simplicity, the metal will be assumed perfectly conducting (assumption valid at frequencies up to far-infrared) and no dielectrics will be included for the first part of the Letter, although they will be taken into account when comparing to experimental results.

In Fig. 2, the modeled dispersion relation of the surface mode supported by the structure is shown for different heights of the notches when the system possesses glide symmetry. The figure shows the degeneracy of the two lowest order modes at the Brillouin zone boundary with non-zero slope, which is the principal characteristic of glide symmetry and whose origin will be studied in the following. This special degeneracy introduced by the symmetry leads to a linearization of the mode dispersion with respect to the single notched system, reducing the frequency dependency of the effective refractive index. In contrast to previous studies in closed systems such as corrugated waveguides [16], the coupling to a mode on 
the lightline causes an anti-crossing, which limits the range of linear dispersion of the upper branch (second mode).
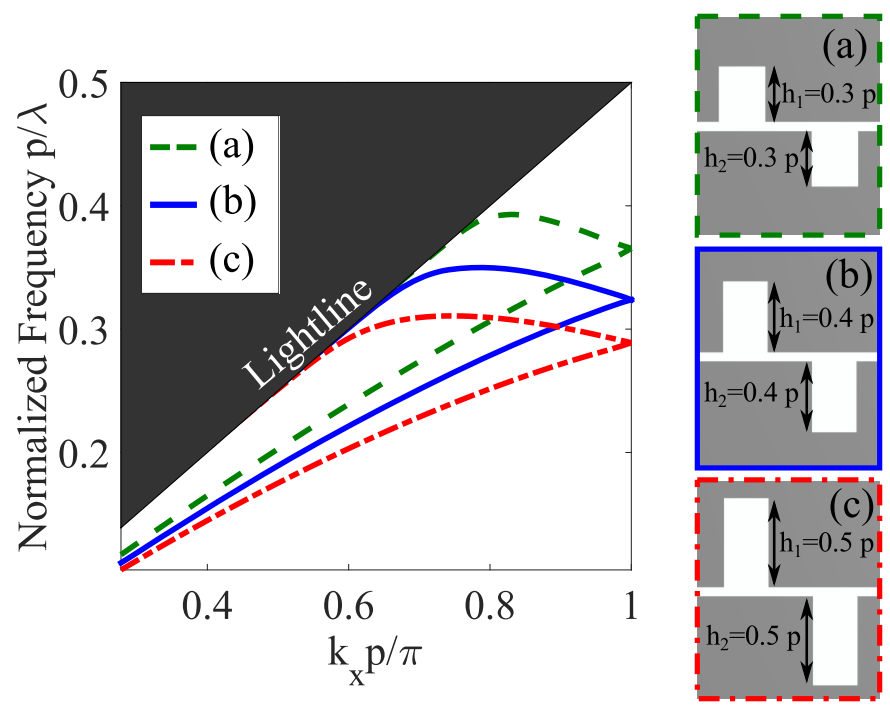

Fig. 2. Predicted dispersion relation for the two lowest order modes of an infinite slot with glide-symmetric notches.

Different heights of the notches corresponding to the different curves are shown in the insets. The relative value of the other geometrical parameters are: $w_{1}=w_{2}=p / 4, g=p / 20$.

In this kind of configuration, by choosing a small slot width (in the case of $w_{1}, w_{2}<p / 2$ ) one can limit the interaction between the fields of adjacent notches due to the requirement of the electric field is transverse to the slot axis. This situation gives a very good insight into the origin of the degeneracy shown in Fig. 2. In the case of non-overlapping sublattices, the plane shown as a dashed line in Fig. 1 can be approximately substituted by a perfect conducting plane thanks to the negligible out-of-plane component of the electric field when compared to the in-plane transverse electric field (y component). This approximation is strictly true when the thickness of the layer tends to infinite, for which the out-ofplane component of the electric field vanishes, although the approximation remains valid for narrow slots. This means that any notch on either side will be mirrored by this conducting plane, and the fields in the system behave equivalently to those in a single-notched unit cell of length $p / 2$. This reduced equivalent unit cell will correspond in terms of $k$ space to a Brillouin zone boundary located at twice the in-plane momentum from the origin, at which the zero group velocity is reached. Therefore, the dispersion of the glide-symmetric configuration is constructed by folding the dispersion relation of the half unit cell system and does not present any band gap.

In Fig. 3, a comparison between the dispersion of the glidesymmetric structure and the case of single notched slot with half the original periodicity are shown, where the two modes are in practice indistinguishable until past the first Brillouin zone boundary of the glide symmetric configuration. The difference at higher frequencies arises from hybridization with a mode on the lightline.

One concludes that glide symmetry allows for the virtual halving of the reciprocal space repeat unit while largely maintaining the field distributions of the modes supported by the non-glided structure. This means that the second lowest order mode in the case of glide symmetry, previously described

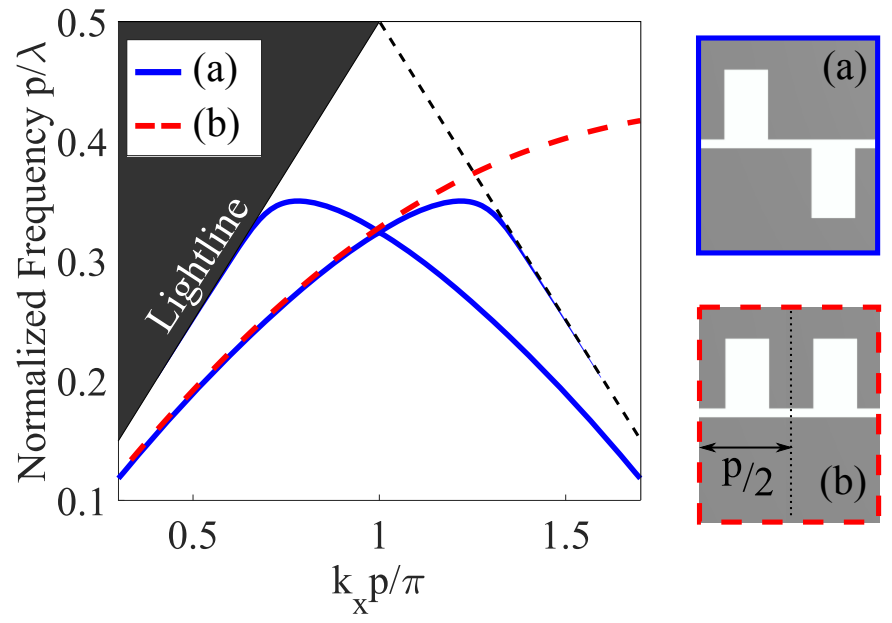

Fig. 3. Comparison between the dispersion relation of the two lowest order modes of the glide-symmetric system (solid blue line) and the lowest order mode of the single notched slot (dashed red line) as shown in the insets for $h_{1}=h_{2}=0.4 p$, $w_{1}=w_{2}=p / 4$ and $g=p / 20$.

as a negative index mode [22], corresponds essentially to the same mode found in the single notched slot system (with positive mode index) in contrast to the truly negative mode index found in mushroom-type metasurfaces [23]. This mode in the glide symmetry case is produced by the backscattering of the original mode into the smaller Brillouin zone of the glide-symmetric system (corresponding to the solid blue curve in Fig 3). This property becomes very important when the distance between two notches (or meta-atoms in general) is small and in practice limited by the precision of the manufacturing process. This limitation can be greatly improved by separately manufacturing the two glided sublattices [21].

We now proceed to model the effects of breaking the glide symmetry of the system.

In Fig. 4, the effect of making the heights of the two notches in the unit cell different is analyzed. Here, it can be seen how the degeneracy with non-zero group velocity is lost, with a band gap appearing at the Brillouin zone boundary. It is interesting to note that the frequency at which each of the two lowest order modes cross the Brillouin zone appears to be largely dependent on the height of only one of the notches. This arises from the concentration of the fields on each sub-lattice. Not surprisingly the linearity of the mode dispersion increases as the notches become similar.

Further modeling shows that, although even small deviations between the sizes of the notches eliminate the degeneracy between the two modes, the linearity of the mode dispersion remains practically unaffected at frequencies below the band-gap. This effect is also present for large deviations (see the red dot-dashed line in Fig. 4) for normalized frequencies below $p / \lambda=0.25$.

In contrast, in Fig. 5 it is shown that when the sizes of the notches are kept the same but the two sub-lattices are no longer at half the unit cell spacing, the two modes again split at the Brillouin zone boundary. This splitting is readily explained by the different field distribution of the two standing waves at the Brillouin zone boundary. The $x$ component of the electric field in the notches are out of phase for the lowest mode and in-phase 

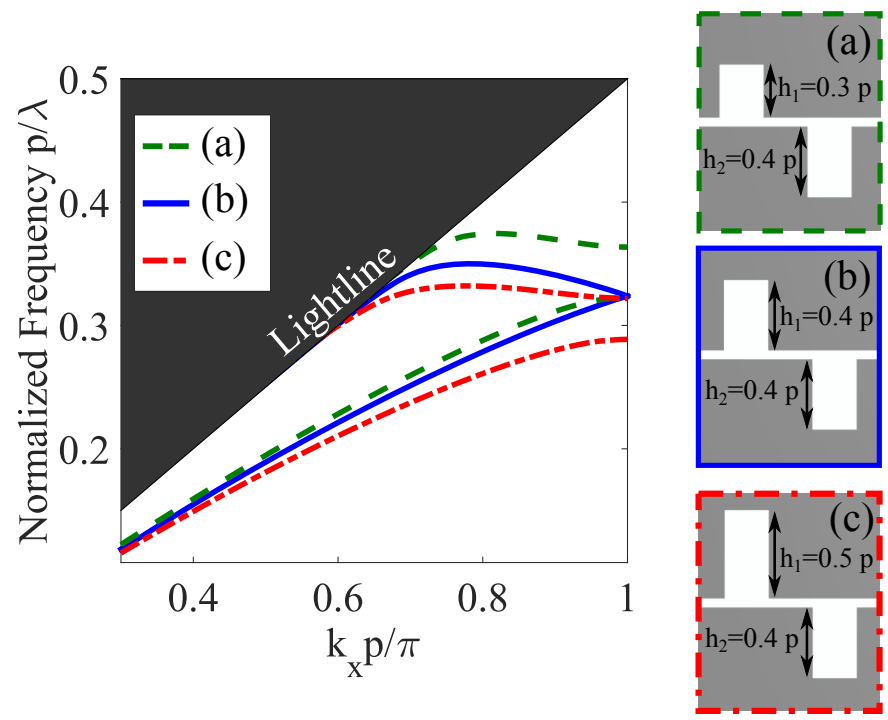

Fig. 4. Predicted dispersion relation of the glide symmetric system (solid blue line) compared to those of the same geometry in which the two notches have different heights, as shown in the insets. The normalized value of the other parameters are $w_{1}=w_{2}=p / 4$ and $g=p / 20$.
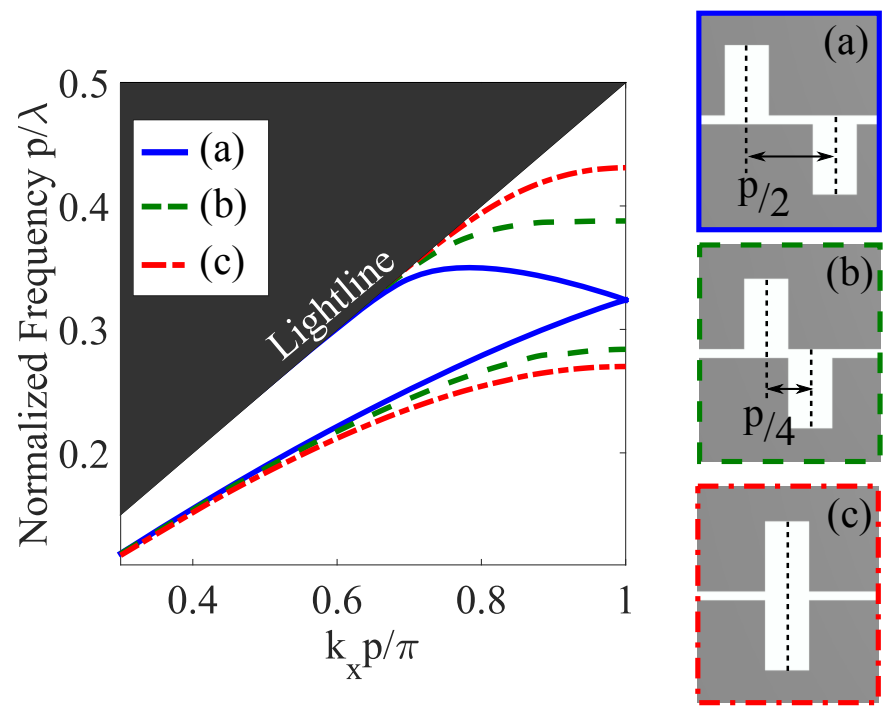

Fig. 5. Dispersion relation of the glide symmetric system (solid blue line) compared to those of the same geometry in which the two notches have been relatively displaced as shown in the insets. The normalized value of the other parameters are $h_{1}=h_{2}=0.4 p, w_{1}=w_{2}=p / 4$ and $g=p / 20$. for the upper mode [22].

Finally, to verify the predictions obtained from a commercial finite-element method software [24], we have undertaken an experiment at microwave frequencies. The sample was manufactured using a print and etch technique applied to a $50 \mu \mathrm{m}$ thick dielectric substrate with dielectric constant value 2.8 coated with a $17 \mu \mathrm{m}$ copper layer (the thickness of the metal can be neglected and it is also treated as perfectly conducting in the modeling). The unit cell was chosen to be $10 \mathrm{~mm}$ and the other parameters had values of $h_{1}=h_{2}=4 \mathrm{~mm}, w_{1}=w_{2}=$ $2.5 \mathrm{~mm}$ and $g=0.5 \mathrm{~mm}$.

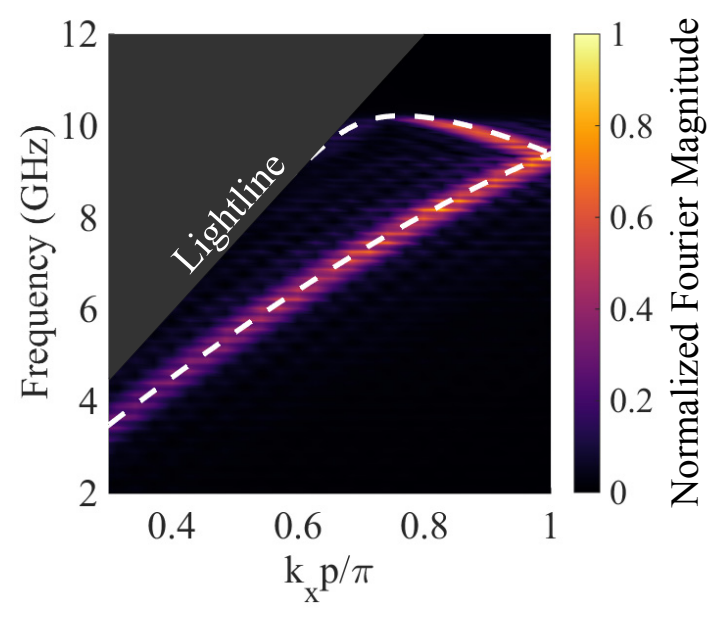

Fig. 6. Experimental and modeled dispersion for the two lowest order modes of the glide symmetric slot structure. The colormap represents the Fourier amplitude obtained from the fast Fourier transform of the measured $y$ electric field component distribution and the superimposed white dashed lines represent the modelled dispersion relation. The values of the parameters are $h_{1}=h_{2}=4 \mathrm{~mm}, w_{1}=w_{2}=2.5 \mathrm{~mm}$, $p=10 \mathrm{~mm}$ and $g=0.5 \mathrm{~mm}$.

The mode of the system was excited by soldering the inner and outer conductors of a coaxial transmission line to either edge of the slot. Then the $y$ component of the electric field was measured by using a modified coaxial antenna placed in the near field of the metasurface. By scanning along the sample and performing a fast Fourier transform from the spatial domain to $k$ space along the direction of propagation, the experimental dispersion shown in Fig. 6 was obtained [25]. Superimposed on the Fourier amplitude of the measured fields, we show the modeled dispersion with the dielectric layer included in the model, obtaining excellent agreement. As predicted by the modeling, we find that the mode at the Brillouin zone boundary has non-zero group velocity, evidenced by the fact that the mode has been detected at the crossing point. Also note that, at the turning point of the second mode near $10 \mathrm{GHz}$, the mode cannot be detected due to the lack of energy propagation when the group velocity approaches zero.

In Fig. 7 the experimentally measured distribution of the $y$ component of the electric field is shown at $1 \mathrm{~mm}$ above the surface and compared to the simulated field distribution. We again find excellent agreement with those predicted by the model, with some residual $z$ component of the electric field being measured on the metal surface.

In conclusion, the surface mode propagation on a single 


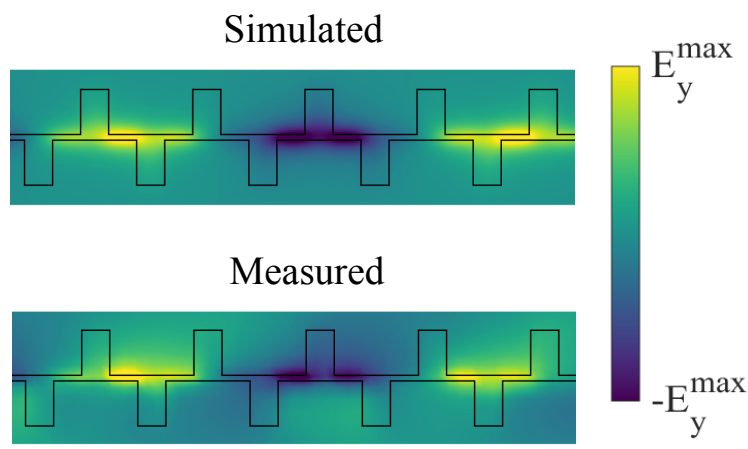

Fig. 7. Modelled and measured $y$ component of the electric field distribution at $z=1 \mathrm{~mm}$ above the surface when exciting the system at $6.5 \mathrm{GHz}$. The values of the parameters are $h_{1}=$ $h_{2}=4 \mathrm{~mm}, w_{1}=w_{2}=2.5 \mathrm{~mm}, p=10 \mathrm{~mm}$ and $g=0.5 \mathrm{~mm}$.

layer glide-symmetric structure formed by an infinitely-long slot with periodic notches on both sides has been studied. Through modeling we have explained the origin of the nonzero group velocity of the mode at the Brillouin zone boundary and the advantages that this symmetry may have when manufacturing highly dense arrays with respect to traditional down scaling. We have explored the effects of breaking the symmetry of the system and explained the appearance of two non-degenerate modes. We have validated these properties by experimentally reproducing the dispersion diagram of a glidesymmetric structure and by characterizing the modeled field distribution on the slot.

The authors wish to acknowledge financial support from the Engineering and Physical Sciences Research Council (EPSRC) of the United Kingdom, via the EPSRC Centre for Doctoral Training in Metamaterials (Grant No. EP/L015331/1). All data created during this research are openly available from the University of Exeter's institutional repository at https://ore.exeter.ac.uk/.

\section{REFERENCES}

1. R. H. Ritchie, Physical Review 106, 874 (1957).

2. W. L. Barnes, A. Dereux, and T. W. Ebbesen, Nature 424, 824 (2003).

3. S. A. Maier, Plasmonics: Fundamentals and applications (Springer US, Boston, MA, 2007).

4. J. B. Pendry, L. Martín-Moreno, and F. J. Garcia-Vidal, Science 305, 847 (2004).

5. A. Hibbins, B. Evans, and J. Sambles, Science 308, 670 (2005).

6. F. J. García De Abajo and J. J. Sáenz, Physical Review Letters 95, 1 (2005).

7. J. A. Dockrey, M. J. Lockyear, S. J. Berry, S. A. R. Horsley, J. R. Sambles, and A. P. Hibbins, Physical Review B 87, 125137 (2013).

8. F. Monticone and A. Alù, Proceedings of the IEEE 103, 793 (2015).

9. X. Shen, T. J. Cui, D. Martin-Cano, and F. J. Garcia-Vidal, Proceedings of the National Academy of Sciences of the United States of America 110, 40 (2013).

10. H. F. Ma, X. Shen, Q. Cheng, W. X. Jiang, and T. J. Cui, Laser and Photonics Reviews 8, 146 (2014).

11. H. Chen, K. K. Tsia, and A. W. Poon, Optics Express 14, 7368 (2006).

12. W. T. Lau and S. Fan, Applied Physics Letters 81, 3915 (2002).
13. M. N. Erim, N. Erim, and H. Kurt, Photonics and Nanostructures Fundamentals and Applications 11, 123 (2013).

14. I. H. Giden, M. Turduev, and H. Kurt, Journal of the European Optical Society 9 (2014).

15. V. R. Almeida, Q. Xu, C. A. Barrios, and M. Lipson, Optics Letters 29 , 1209 (2004).

16. G. Valerio, Z. Sipus, A. Grbic, and O. Quevedo-Teruel, IEEE Transactions on Antennas and Propagation 65, 2695 (2017).

17. R. C. Mitchell-Thomas, I. R. Hooper, J. R. Sambles, A. P. Hibbins, and O. Quevedo-Teruel, "Broadband metasurface for surface wave lenses," in "2016 URSI International Symposium on Electromagnetic Theory (EMTS)," (IEEE, 2016), pp. 605-606.

18. P. Crepeau and P. Mclsaac, Proceedings of the IEEE 52, 33 (1964).

19. A. Hessel, Ming Hui Chen, R. Li, and A. Oliner, Proceedings of the IEEE 61, 183 (1973).

20. O. Quevedo-Teruel, M. Ebrahimpouri, and M. Ng Mou Kehn, IEEE Antennas and Wireless Propagation Letters 15, 484 (2016).

21. M. Ebrahimpouri, E. Rajo-Iglesias, Z. Sipus, and O. QuevedoTeruel, "Low-cost metasurface using glide symmetry for integrated waveguides," in "2016 10th European Conference on Antennas and Propagation, EuCAP 2016," (IEEE, 2016), pp. 1-2.

22. R. Quesada, D. Martín-Cano, F. J. García-Vidal, and J. Bravo-Abad, Optics Letters 39, 2990 (2014).

23. J. A. Dockrey, S. A. R. Horsley, I. R. Hooper, J. R. Sambles, and A. P. Hibbins, Scientific Reports 6, 22018 (2016).

24. "CST Microwave Studio," http://www.cst.com.

25. M. Camacho, R. R. Boix, F. Medina, A. P. Hibbins, and J. R. Sambles, Physical Review B 95, 245425 (2017). 


\section{FULL REFERENCES}

1. R. H. Ritchie, "Plasma Losses by Fast Electrons in Thin Films," Physical Review 106, 874-881 (1957).

2. W. L. Barnes, A. Dereux, and T. W. Ebbesen, "Surface plasmon subwavelength optics," Nature 424, 824-830 (2003).

3. S. A. Maier, Plasmonics: Fundamentals and applications (Springer US, Boston, MA, 2007).

4. J. B. Pendry, L. Martín-Moreno, and F. J. Garcia-Vidal, "Mimicking surface plasmons with structured surfaces." Science 305, 847-8 (2004).

5. A. Hibbins, B. Evans, and J. Sambles, "Experimental verification of designer surface plasmons," Science 308, 670-672 (2005).

6. F. J. García De Abajo and J. J. Sáenz, "Electromagnetic surface modes in structured perfect-conductor surfaces," Physical Review Letters 95, 1-4 (2005).

7. J. A. Dockrey, M. J. Lockyear, S. J. Berry, S. A. R. Horsley, J. R. Sambles, and A. P. Hibbins, "Thin metamaterial Luneburg lens for surface waves," Physical Review B 87, 125137 (2013).

8. F. Monticone and A. Alù, "Leaky-wave theory, techniques, and applications: From microwaves to visible frequencies," Proceedings of the IEEE 103, 793-821 (2015).

9. X. Shen, T. J. Cui, D. Martin-Cano, and F. J. Garcia-Vidal, "Conformal surface plasmons propagating on ultrathin and flexible films." Proceedings of the National Academy of Sciences of the United States of America 110, 40-5 (2013).

10. H. F. Ma, X. Shen, Q. Cheng, W. X. Jiang, and T. J. Cui, "Broadband and high-efficiency conversion from guided waves to spoof surface plasmon polaritons," Laser and Photonics Reviews 8, 146-151 (2014).

11. H. Chen, K. K. Tsia, and A. W. Poon, "Surface modes in twodimensional photonic crystal slabs with a flat dielectric margin," Optics Express 14, 7368 (2006).

12. W. T. Lau and S. Fan, "Creating large bandwidth line defects by embedding dielectric waveguides into photonic crystal slabs," Applied Physics Letters 81, 3915-3917 (2002).

13. M. N. Erim, N. Erim, and H. Kurt, "Optical surface modes of photonic crystals for dual-polarization waveguide," Photonics and Nanostructures - Fundamentals and Applications 11, 123-131 (2013).

14. I. H. Giden, M. Turduev, and H. Kurt, "Reduced symmetry and analogy to chirality in periodic dielectric media," Journal of the European Optical Society 9 (2014).

15. V. R. Almeida, Q. Xu, C. A. Barrios, and M. Lipson, "Guiding and confining light in void nanostructure," Optics Letters 29, 1209 (2004).

16. G. Valerio, Z. Sipus, A. Grbic, and O. Quevedo-Teruel, "Accurate Equivalent-Circuit Descriptions of Thin Glide-Symmetric Corrugated Metasurfaces," IEEE Transactions on Antennas and Propagation 65, 2695-2700 (2017).

17. R. C. Mitchell-Thomas, I. R. Hooper, J. R. Sambles, A. P. Hibbins, and O. Quevedo-Teruel, "Broadband metasurface for surface wave lenses," in "2016 URSI International Symposium on Electromagnetic Theory (EMTS)," (IEEE, 2016), pp. 605-606.

18. P. Crepeau and P. Mclsaac, "Consequences of symmetry in periodic structures," Proceedings of the IEEE 52, 33-43 (1964).

19. A. Hessel, Ming Hui Chen, R. Li, and A. Oliner, "Propagation in periodically loaded waveguides with higher symmetries," Proceedings of the IEEE 61, 183-195 (1973).

20. O. Quevedo-Teruel, M. Ebrahimpouri, and M. Ng Mou Kehn, "Ultrawideband Metasurface Lenses Based on Off-Shifted Opposite Layers," IEEE Antennas and Wireless Propagation Letters 15, 484487 (2016).

21. M. Ebrahimpouri, E. Rajo-Iglesias, Z. Sipus, and O. QuevedoTeruel, "Low-cost metasurface using glide symmetry for integrated waveguides," in "2016 10th European Conference on Antennas and Propagation, EuCAP 2016," (IEEE, 2016), pp. 1-2.

22. R. Quesada, D. Martín-Cano, F. J. García-Vidal, and J. BravoAbad, "Deep-subwavelength negative-index waveguiding enabled by coupled conformal surface plasmons," Optics Letters 39, 2990 (2014).

23. J. A. Dockrey, S. A. R. Horsley, I. R. Hooper, J. R. Sambles, and A. P. Hibbins, "Direct observation of negative-index microwave surface waves," Scientific Reports 6, 22018 (2016).

24. "CST Microwave Studio," http://www.cst.com.

25. M. Camacho, R. R. Boix, F. Medina, A. P. Hibbins, and J. R. Sambles, "Theoretical and experimental exploration of finite sample size effects on the propagation of surface waves supported by slot arrays," Physical Review B 95, 245425 (2017). 